\title{
LE CAPITALISME FAMILIAL \\ DANS LES ENTREPRISES MOYENNES : \\ UN DÉCLIN RÉVERSIBLE
}

\author{
par Hervé JOLY \\ Centre Pierre Léon
}

Ni l'origine familiale des moyennes entreprises ni leur évolution vers un mode de contrôle managérial ne sont une fatalité. Le suivi du contrôle des moyennes entreprises de la région lyonnaise dans la première moitié du $X X^{e}$ siècle vérifie la diversité de l'évolution adoptée par ces firmes.

Comment évolue la propriété du capital des entreprises au fil de leur histoire, dans quelle mesure détermine-t-elle la dévolution des fonctions dirigeantes? Ces importantes questions n'ont à l'évidence pas été réglées de manière définitive par le modèle évolutionniste exclusif présenté par des auteurs comme Berle et Means ${ }^{(1)}$ ou Burnham ${ }^{(2)}$. Selon la vision aujourd'hui communément répandue qu'ils ont inspirée, le capital d'une entreprise, concentré au début entre les mains du fondateur et éventuellement de ses descendants, serait ensuite progressivement ouvert jusqu'à une dilution complète dans l'anonymat d'actionnaires dispersés; la direction, émancipée de la propriété, reviendrait alors à des «managers » recrutés pour leurs seules compétences. Il convient de s'interroger sur la représentativité réelle de ce modèle. À quel rythme s'effectue cette évolution? Est-elle nécessairement aussi linéaire et irréversible?

Ces questions paraissent particulièrement intéressantes pour des entreprises restées moyennes. Les petites entreprises, simple extension d'activités artisanales, restent à l'évidence le domaine de la propriété personnelle ou familiale. Les grandes entreprises, qui ont fait l'objet de nombreuses monographies, illustrent largement

(1) A.A. Berle Jr., G.C. Means, The Modern Corporation and Private Property, New York, MacMillan, 1932.

(2) J. Burnham, L'ère des organisateurs, Paris, Calmann-Lévy, 1947. 
ce schéma ${ }^{(3)}$. L'emprise familiale y atteint effectivement, dans un temps plus ou moins long, ses limites : 1'appel à des capitaux et à des compétences dirigeantes extérieurs devient, à un moment ou un autre, indispensable pour assurer le développement. La situation des entreprises de l'échelle intermédiaire mérite en revanche d'être examinée de plus près.

L'étude doit porter sur un corpus assez large pour éviter les aléas de la monographie. Le nombre d'entreprises étudiées ne peut cependant, dans le cadre de cet article, dépasser plusieurs dizaines : répondre aux questions posées implique de rassembler de multiples informations, nécessairement dispersées, sur les entreprises et leurs dirigeants. La seule utilisation du registre du commerce, effectuée dans le cadre d'une large étude régionale portant sur les modes de contrôle d'un millier d'entreprises et d'établissements de plus de 100 salariés, s'avère insuffisante pour des analyses fines ${ }^{(4)}$. La consultation de nombreux actes de sociétés, dont les collections sont - avec un classement chronologique de l'ensemble des actes déposés dans un même greffe longtemps restées dispersées ${ }^{(5)}$, est diffici$1 \mathrm{e}^{(6)}$. L'étude s'est donc limitée à des entreprises moyennes (entre 100 et 500 salariés $\left.{ }^{(7)}\right)$, ayant eu leur siège dans le ressort du tribunal de commerce de Lyon (arrondissement de Lyon $)^{(8)}$. Il est particulièrement intéressant d'observer le devenir de ces nouvelles activités industrielles dont Pierre Cayez avait décrit le fort développement, à côté des activités traditionnelles de la soierie, dans l'agglomération au cours de la deuxième moitié du XIX Siècle $^{(9)}$. Les principales branches caractéristiques de cette seconde industrialisation ont été retenues : la construction mécanique, la chimie, et, plus tardivement, la construction électrique $^{(10)}$. Un corpus de 60 entreprises (répar-

(3) Pour une synthèse sur le cas des grandes entreprises françaises et anglaises, cf. F. Crouzet, « Les dynasties d'entrepreneurs en France et en Grande-Bretagne », Entreprises et Histoire, 1995, n 9, p. 25-42. Sur le cas de l'Allemagne, cf. H. Joly, Patrons d'Allemagne. Sociologie d'une élite industrielle. 1933-1989, Paris, Presses de Sciences Po, 1996, p. 25-65.

(4) Les résultats de cette étude sont présentés dans un rapport de recherche remis, avec F. Robert (ingénieur CNRS au Centre Pierre Léon), à l'Agence Rhône-Alpes pour les Sciences sociales et humaines en mai 2001, Le contrôle économique des entreprises dans la région Rhône-Alpes (1920-1954). Implantation des centres de décision économique et bassins d'emploi régionaux, 2 volumes (à paraître sous forme de livre en 2002 à Lyon aux Éditions de l'ISH).

(5) Ce n'est qu'après la mise en place du nouveau registre du commerce en 1954 que les actes sont regroupés par société.

(6) Elle l'est d'autant plus, sauf arrangement particulier avec les conservateurs, lorsque les actes sont déposés aux archives départementales : la consultation de chaque acte, qui peut parfois être très succinct, est comptabilisée dans le nombre limité de communications accordées par jour.

(7) Il s'agit plus précisément d'entreprises dont la somme des effectifs maximaux des différents établissements dans les huit départements de la région Rhône-Alpes connus pour avoir atteint 100 salariés entre 1936 et 1949 , d'après les différentes sources disponibles pour l'époque (conflits du travail, délégation régionale du ministère de la Production industrielle, entre autres, aux Archives départementales du Rhône ; fichier des établissements de l'Office central de répartition des produits industriels aux Archives nationales), ne dépasse pas 500 salariés.

(8) Ont été retenues les entreprises ayant eu, en tant que société, leur premier siège dans l'arrondissement de Lyon et qui l'avaient encore en 1936, date à partir de laquelle les effectifs sont pris en compte, et qui avaient au moins un établissement dans cet arrondissement.

(9) P. Cayez, Crises et croissance de l'industrie lyonnaise : 1850-1900, Paris, CNRS Éditions, 1980.

(10) Selon le code à deux ou trois chiffres adopté par l'INSEE en 1949 : codes 20 à 27, 29-2 (précision, horlogerie et optique), 33-4 (charpentes et constructions métalliques) et 56 (bijouterie, orfèvrerie, joaillerie) pour la transformation de l'acier et la construction mécanique au sens large, 28 pour la construction électrique, 35,36 et 37 pour les industries chimiques, le caoutchouc et l'amiante. 
ties entre 34,13 et 13 respectivement dans les trois branches) a ainsi été constitué(11). Aux inscriptions, éventuellement successives en cas de changement de statut, de ces entreprises dans le premier registre de commerce de Lyon (1920-1954) déjà relevées pour l'étude régionale s'est ajoutée la consultation aussi systématique que possible des actes de sociétés depuis les débuts de l'exploitation en société, éventuellement antérieure à 1920. Enfin, pour la connaissance plus précise des dirigeants et de leurs structures familiales, des sources classiques d'histoire sociale (listes mondaines de l'Annuaire Tout Lyon, registres d'état civil, déclarations de succession, listes nominatives du recensement, listes électorales à la Chambre de commerce, etc.) ont été, dans la limite des délais de consultation à laquelle certaines d'entre elles sont soumises, utilisées à titre complémentaire ${ }^{(12)}$.

Le modèle évolutionniste apparaît largement confirmé par l'évolution des statuts des entreprises étudiées dans le cadre chronologique retenu (cf. tableau 1). On passe bien d'un capital très concentré, avec une prédominance de sociétés de personnes (16) - sociétés en nom collectif (SNC) ou en commandite simple (SCS) - et surtout d'entreprises individuelles $^{(13)}$ (27) au début, à un capital tendant à devenir anonyme (43 sociétés anonymes $\left.-\mathrm{SA}^{(14)}\right)$ à la fin de la période. Lors de la première inscription dans le registre du commerce, les sociétés par actions sont peu nombreuses (16) et elles sont d'ailleurs presque toutes de formation récente (4 seulement avant 1914) ; la période de l'immédiat après-guerre est notamment très favorable à l'adoption de ce statut. Ces entreprises auraient donc seulement quelques années d'avance sur les autres dans leur développement. À l'inverse, en 1954, les statuts à capital fermé ont presque entièrement disparu, sous réserve cependant de l'émergence, à partir de son instauration en 1925, du statut de la société à responsabilité limitée (SARL), qui apparaît comme une forme hybride: elle emprunte à la société par actions le nombre en théorie illimité d'associés et l'engagement financier réduit à leur apport, mais, comme la société de personnes, elle n'autorise pas la mise en souscription publique. En pratique, le nombre d'associés reste réduit dans la mesure où les parts ne peuvent pas être cédées sur le marché. Le « triomphe de la SARL » ${ }^{(15)}$, relatif en l'occurrence pour des entreprises de cette taille, s'est surtout fait aux dépens des entreprises individuelles et des sociétés de personnes, mais les chiffres agrégés cachent des évolutions plus contrastées. Alors qu'un quart des entreprises (15) n'ont pas changé de statut entre 1920 et 1954, plus de deux cinquièmes (25) en ont changé au moins deux fois. Si la plupart des changements se font dans le sens d'une ouverture croissante (commerçant, SNC, puis SA ou SNC, SARL puis SA par exemple), on relève toutefois six cas d'évolutions en sens inverse, de la SA à la SARL,

(11) En pratique, toutes ces entreprises sauf deux (qui ont une usine dans les deux villes voisines de Lyon et de Villeurbanne) n'ont qu'un seul établissement dans le corpus régional des établissements de 100 salariés au moins. Une seule est connue, d'après le fichier de l'OCRPI, pour avoir un autre établissement entre 50 et 99 salariés dans la région, trois en ayant au moins un de plus de 50 salariés ailleurs en France. Le corpus est donc dominé, de manière écrasante, par des entreprises « mono-établissements », dont le siège social se trouve confondu avec l'implantation industrielle unique, ce qui correspond bien au modèle de l'entreprise moyenne.

(12) Pour alléger les notes, les références de toutes ces sources ne seront pas systématiquement indiquées dans l'article.

(13) Les entreprises individuelles sont inscrites dans la série A du registre du commerce avec un commerçant unique (plusieurs commerçants inscrits pour la même entreprise seraient en société de personnes de fait).

(14) Jusqu'en 1931, il existe aussi, parmi les sociétés par actions, une société en commandite par actions.

(15) F. Caron, J. Bouvier, in F. Braudel, E. Labrousse (dir.), Histoire économique et sociale de la France, tome IV : 1880-1950, Paris, PUF, Quadrige, 1993, p. 774. 
Tableau 1 : Évolution de la répartition des statuts des entreprises du corpus étudié

\begin{tabular}{|l|c|c|c|c|c|}
\hline \multicolumn{1}{|c|}{ Statuts } & $\begin{array}{c}\text { À la 1e inscription } \\
\text { au registre } \\
\text { du commerce } \\
\text { (1920 ou après) }\end{array}$ & $\begin{array}{c}\text { Au 1er janvier } \\
1931\end{array}$ & $\begin{array}{c}\text { Au 1er janvier } \\
1941\end{array}$ & $\begin{array}{c}\text { Au 1er janvier } \\
1951\end{array}$ & $\begin{array}{c}\text { Dernière mention } \\
\text { dans le registre } \\
\text { du commerce } \\
\text { (1954 ou avant) }\end{array}$ \\
\hline $\begin{array}{l}\text { Entreprise } \\
\text { individuelle }\end{array}$ & 27 & 14 & 10 & 2 & 2 \\
\hline $\begin{array}{l}\text { Sociétés } \\
\text { de personnes }\end{array}$ & 16 & 11 & 3 & 0 & 0 \\
\hline $\begin{array}{l}\text { SARL } \\
\text { (à partir de 1925) }\end{array}$ & $1^{117)}$ & 8 & 11 & 18 & 15 \\
\hline $\begin{array}{l}\text { Sociétés } \\
\text { par actions }\end{array}$ & 16 & 26 & 35 & 38 & 43 \\
\hline Total & 60 & $59^{(18)}$ & $59^{(19)}$ & $58^{201}$ & 60 \\
\hline
\end{tabular}

définitives (3) ou provisoires (3). Une SA qui, faute d'appel au public, a un faible nombre d'actionnaires est susceptible de (re)devenir une SARL. La cotation en bourse reste en effet exceptionnelle dans un corpus d'entreprises de cette taille: 7 seulement l'ont été au cours de la période, dont une à titre provisoire ${ }^{(16)}$. La transformation en société par actions ne semble donc pas nécessairement impliquer une dilution du capital.

Le modèle évolutionniste, avec une entreprise passée de l'exploitation sous la forme individuelle par son fondateur à une gestion, dans le cadre d'une société par actions au capital ouvert, par de simples mandataires, souffre d'importantes exceptions. D'un côté, toutes les entreprises ne relèvent pas de l'initiative originelle d'un entrepreneur unique et, vice versa, le contrôle individuel ne correspond pas forcément à une situation originelle. D'un autre côté, à la fin de la période du moins, l'éventuelle ouverture du capital à l'extérieur n'entraîne pas une dissociation entre propriété individuelle et pouvoir ; elle n'exclut pas le maintien d'une importante dimension familiale, fondatrice d'origine ou non.

\section{DES ALTERNATIVES À LA CRÉATION PAR UN ENTREPRENEUR UNIQUE}

D'une part, toute entreprise ne repose pas sur l'initiative isolée d'un entrepreneur complet, éventuellement inventeur, au moins fabricant et commerçant. Elle peut être créée dès le départ sous la forme d'une société entre deux ou trois personnes qui sont autant de fondateurs. Ils trouvent une complémentarité dans leur association : un industriel et un négociant, un inventeur de procédé et un apporteur de capitaux, deux

(16) Ce recensement devrait être à peu près exhaustif dans la mesure où il repose sur le dépouillement de diverses éditions d'annuaires de sociétés, et en particulier celui des sociétés cotées à la Bourse de Lyon.
(17) Création en 1934.
(18) Une création ultérieure.
(19) Une disparition antérieure.
(20) Deux disparitions antérieures. 
ingénieurs détenteurs d'une technologie commune. L'entreprise d'appareillage électro-industriel Pétrier, Tissot \& Raybaud associe ainsi, lors de sa création sous la forme d'une SNC en 1909, deux industriels du même âge (39 ans) et de la même origine géographique (la Haute-Savoie) ${ }^{(21)}$. Ils sont rejoints quelques années plus tard par un ingénieur de 7 ans leur cadet, originaire des Alpes-Maritimes. Lorsque le capital, déjà divisé en trois tiers, est ouvert, après la transformation en SA en 1918 suivie de la cotation en bourse en 1921, les fondateurs et leurs familles se retrouvent rapidement en position minoritaire. En 1928, ils adoptent la technique des actions à droit de vote plural pour préserver leur indépendance. En 1931, ils apportent encore $47 \%$ des capitaux parmi 231 souscripteurs mais, en 1949, leur part tombe à $18 \%$ parmi 1221 souscripteurs.

La création immédiate d'une entreprise sous la forme d'une société par actions concerne traditionnellement les activités qui ne connaissent pas, par nature, un stade préalable quasi-artisanal et qui requièrent d'emblée des investissements importants. C'est le cas, en particulier, des compagnies de transport (navigation, chemins de fer), d'exploitation minière, de distribution de gaz puis d'électricité(22). L'hypothèse est logiquement moins fréquente parmi les entreprises du corpus (6 sur 60 seulement), à l'exception de la construction électrique à la hauteur de près d'un tiers (4 sur 13$)^{(23)}$. Une SA comprend un minimum légal de sept associés. Même si l'un d'entre eux peut concentrer la quasi-totalité du capital entre ses mains, le recours à ce statut s'accompagne généralement d'un appel à des investisseurs extérieurs, au-delà du ou des fondateurs directement engagés dans l'affaire. En 1919 , les quatre fondateurs de l'entreprise de câblerie Le Fil Dynamo, deux ingénieurs de l'École supérieure d'électricité et deux contremaîtres, ne détiennent ainsi qu'un quart du capital de leur nouvelle $\mathrm{SA}^{(24)}$. Leurs apports en nature, sous la forme d'études pour la constitution de la société, de procédés de fabrication, de relations commerciales et professionnelles, etc., ne représentent que $10 \%$ du capital et ils ne souscrivent par ailleurs que $15 \%$ en numéraire, une contribution de la mère de l'ingénieur André Locquin, veuve d'un avocat de Nevers, ajoutant $8 \%$ supplémentaires. La majorité du capital se trouve donc entre les mains de vingt-cinq investisseurs extérieurs, au sein desquels dominent des industriels ou négociants lyonnais de la métallurgie. Au conseil d'administration, les quatre fondateurs, administrateurs statutaires, doivent, dès l'assemblée générale constitutive, faire la place à quatre autres actionnaires.

La création en 1927 de la Société lyonnaise pour l'exploitation de véhicules électriques (SLEVE) correspond, elle, au modèle classique des compagnies électriques ${ }^{(25)}$. L'entreprise est d'ailleurs à l'origine une quasi-filiale de la Cie du Gaz de Lyon $^{(26)}$ : son directeur général est le fondateur de la SA, dépositaire des statuts, et elle souscrit près de la moitié des actions (45\%). Le reste du capi-

(21) En l'absence d'autres informations, l'origine géographique est ici entendue comme le lieu de naissance indiqué par le registre du commerce.

(22) P. Cayez, Métiers Jacquard et hauts fourneaux. Aux origines de l'industrie lyonnaise, Lyon, PUL, 1978, p. 255.

(23) Les deux autres sont des entreprises de la première transformation de l'acier, les Aciéries du Rhône et les Fonderies et ateliers du Rhône, qui requièrent également des investissements initiaux relativement importants.

(24) Acte de formation déposé le 23 juin 1919 au Tribunal de commerce (TC) de Lyon ; Archives départementales du Rhône (ADR), 6U.

(25) Acte 13 septembre 1927, TC Lyon ; ADR, 6U.

(26) Cette création intervient un an avant que la Cie du Gaz de Lyon ne passe sous le contrôle du groupe Durand ; C. Vuillermot, Pierre-Marie Durand et l'Energie industrielle. L'histoire d'un groupe électrique 1906-1945, Paris, CNRS Éditions, 2001, p. 82-83. 
Figure 1 : Répartition des modes de contrôle des entreprises du corpus

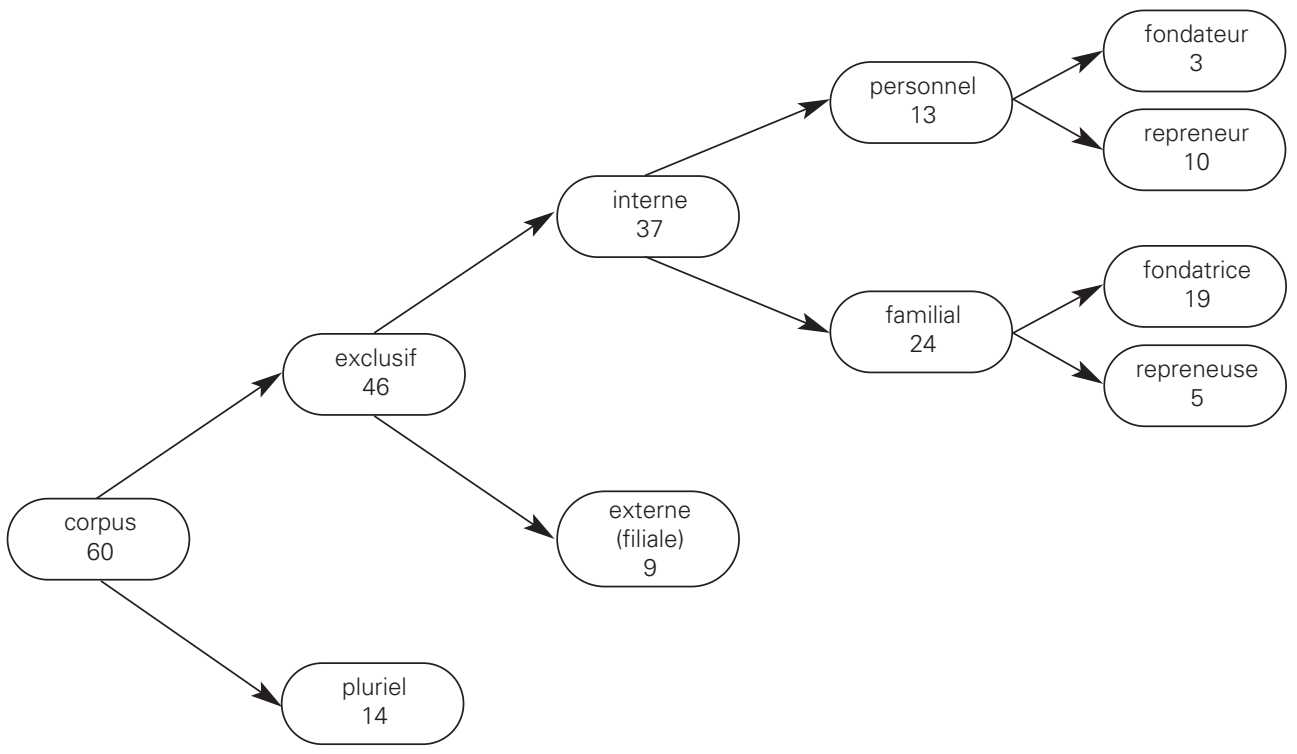

tal est apporté, pour l'essentiel, par d'autres entreprises de production-distribution de gaz et d'électricité (Société lyonnaise des forces motrices du Rhône, Compagnie hydroélectrique d'Auvergne...), de construction électrique (Compagnie des compteurs, MerlinGerin...) ou automobile (Renault). À l'exception de l'unique action de garantie détenue par les administrateurs, cette entreprise n'a pas directement d'actionnaires individuels et se trouve immédiatement sous un contrôle de type managérial (figure 1).

\section{UN CONTRÔLE INDIVIDUEL QUI NE CORRESPOND PAS FORCÉMENT À UNE SITUATION ORIGINELLE}

Parmi les 60 entreprises du corpus, près d'un quart (13) relèvent encore, à la fin de la période étudiée, d'un contrôle individuel, soit, de manière absolue, par un propriétaire unique (2 cas), soit, de manière dominante, par un actionnaire majoritaire d'une société (2 SARL et 9 SA). Mais seuls trois de ces «contrôleurs» peuvent être considérés comme les fondateurs. Les autres sont des « repreneurs » qui ont, à un moment ultérieur, pris le contrôle de l'entreprise, dans différentes situations possibles. Un fonds d'entreprise individuelle peut d'abord être vendu à un repreneur non apparenté. Un dénommé Demont a ainsi cédé, en 1923, à Paul Millier, 33 ans, son fonds de construction mécanique (Ets P. Millier). Il ne s'agit pas d'une succession naturelle : le vendeur n'a que six ans de plus que l'acquéreur. L'activité de fabrication de pompes est réorientée vers celle d'instruments de pesage. La transaction s'est probablement effectuée à un moment où l'entreprise n'avait pas atteint un stade industriel ${ }^{(27)}$, à un prix abor-

(27) La première information disponible sur l'effectif date de 1938, avec 120 salariés. 
dable pour un nouveau venu dans les affaires. L'opération peut même se répéter à plusieurs reprises. Les laboratoires de produits pharmaceutiques sous licence CIBA ont ainsi appartenu à trois pharmaciens successifs. Après avoir racheté le fonds en 1913 au prix de 40000 F, Jules Oscar Rolland l'a revendu, à l'âge de 74 ans, en 1941, à son jeune confrère Paul Denoyel, 36 ans, pour plus de 13 millions de francs ${ }^{(28)}$. Il est probable ici que l'absence de transmission familiale s'explique par les contraintes législatives qui réservent la direction d'une entreprise pharmaceutique à des pharmaciens diplômés ${ }^{(29)}$.

Autre hypothèse, une personne extérieure à la famille du fondateur devient associée de son entreprise, dont elle prend ensuite, par rachat de parts, le contrôle majoritaire. Alphonse Lambert a ainsi apporté en 1935, à l'âge de 59 ans, le fonds d'une entreprise d'équipements de cycles, qu'il avait fondée, à une SARL, avec François Cagnon, de 7 ans son cadet, comme co-gérant ${ }^{(30)}$. À la suite de deux autres cessions de parts en 1936 et 1937, les deux partenaires se retrouvent à parité. Après le décès de Lambert en 1940, sa veuve le remplace comme co-gérante, mais Cagnon prend la majorité du capital. Ce fils d'épicier n'a eu à faire que des investissements financiers relativement modestes, rapidement couverts par ses revenus, pour y parvenir. Alors que le fondateur n'était plus tenu, d'après les statuts de la SARL, qu'à donner à l'entreprise le soin qu'il jugeait nécessaire, Cagnon devait, lui, s'y consacrer à plein temps. En compensation, il a bénéficié, outre un traitement mensuel de $5000 \mathrm{~F}$, d'une répartition des bénéfices supérieure à la proportion de parts qu'il détenait avant d'atteindre la parité. L'allocation d'environ la moitié de bénéfices annuels qui, avant 1935, atteignait déjà près de $300000 \mathrm{~F}$ a dû couvrir l'apport, en trois échéances, d'une somme totale de 500000 F. Il semble bénéficier du fait que le fondateur n'a pas d'enfants. Dans d'autres cas, ceux-ci sont trop jeunes, pas intéressés ou jugés inaptes à la gestion de l'entreprise. "L'héritier de rechange » est généralement un fidèle collaborateur salarié ${ }^{(31)}$.

Dans une troisième hypothèse, un actionnaire de l'entreprise, au départ dans une position minoritaire au sein d'un capital éclaté, en prend ultérieurement le contrôle majoritaire. Il utilise généralement les conditions avantageuses que lui procure sa position de dirigeant ; grâce à la confiance du conseil d'administration, il investit ainsi les revenus qu'il s'attribue dans des augmentations de capital qu'il organise. L'ingénieur des Arts et Métiers et de l'École supérieure d'électricité de Grenoble Léo Trouilhet n'a pas participé directement à la fondation de l'entreprise d'appareils électrodomestiques Calor sous la forme d'une SA en 1917, mais il en est rapidement devenu, comme administrateur délégué, le véri-

(28) Informations données dans l'acte de formation ultérieure, par Paul Denoyel, d'une SARL ; 18 juin 1947, TC Lyon ; ADR, 6U. L'écart considérable de prix, bien supérieur à l'inflation qui n'aurait multiplié le prix que par dix environ, doit s'expliquer par le fait que l'entreprise est, dans l'intervalle, passée d'une simple activité commerciale à la fabrication. Les sources consultées ne permettent pas de savoir comment P. Denoyel, docteur en médecine et en pharmacie, a pu disposer d'une telle somme. Son acte de naissance n'étant pas consultable (né en 1905), son origine sociale (profession des parents) n'est pas connue. Il n'est pas non plus possible de vérifier s'il n'agirait pas comme simple prête-nom de CIBA.

(29) S. Chauveau, L'invention pharmaceutique. La pharmacie française entre l'État et la société au XX siècle, Paris, Institut d'édition Sanofi-Synthélabo, 1999.

(30) Acte $1^{\text {er }}$ octobre 1935, TC Lyon; ADR, 6U.

(31) Dans le cas de F. Cagnon, c'est seulement une hypothèse à confirmer. L'acte de formation de la SARL n'indique pas de profession et il ne figure pas à l'adresse indiquée dans la liste nominative du précédent recensement, en 1931. 
table patron $^{(32)}$. Dès 1918 , il participe, à un niveau modeste (22 actions nouvelles sur 400), à la première souscription nouvelle. À la suivante, en 1920, il est déjà le deuxième souscripteur, avec 103 actions sur 800, derrière le cofondateur Gaston Duveau, également ingénieur. Un an après, les deux mêmes se partagent 600 nouvelles actions, en rémunération d'apports en nature (licences de brevets, inventions et marques de fabrique). Lors des souscriptions suivantes, intervenues à la fin des années 1930 , la position dominante de Léo Trouilhet au capital apparaît : il acquiert, à trois reprises, plus de la moitié des nouvelles actions, avec la seule banque Pictet \& Cie de Genève comme autre souscripteur important ${ }^{(33)}$. Ces augmentations, réalisées au pair ${ }^{(34)}$, qui ont entraîné un quasi-triplement du capital (porté de 1,2 à 3 millions), n'ont représenté pour lui qu'un engagement financier relativement modeste (à peine plus d'un million de francs étalés sur 3 ans) au regard de l'enjeu : une entreprise en forte croissance (plus de 450 salariés en 1946), dont le capital n'est plus augmenté ensuite que sur réserves, est devenue son affaire personnelle. En 1940, il en devient, à 59 ans, un PDG tout puissant, son frère puis son fils siégeant même comme administrateurs à ses côtés.

La dernière hypothèse rencontrée est celle de la prise de contrôle personnel de l'entreprise par un investisseur extérieur, qui n'en était pas dirigeant auparavant. Ce sont généralement des personnalités déjà établies qui réalisent de tels investissements. En 1938, Camille Pras, associé gérant des Ets Tissot, Curis, Pras \& Lombard (Entreprises électriques) ${ }^{(35)}$, souscrit ainsi en numéraires $59 \%$ du capital d'une nouvelle SA des Ateliers rhodaniens ; l'apport en nature de l'ancienne société en commandite simple Isaac \& Cie n'est estimé qu'à $300000 \mathrm{~F}$, soit 37,5\% du nouveau capital, par rapport à un ancien nominal de $750000 \mathrm{~F}^{(36)}$. À la faveur d'une mauvaise santé financière, l'investisseur prend, à bon marché, le contrôle personnel d'une manufacture de tôlerie qui emploie jusqu'à 104 salariés à Villeurbanne en 1943. L'ancien gérant et associé majoritaire, l'ingénieur des Arts et manufactures Jean $\mathrm{Isaac}^{(37)}$, reste cependant à la tête de la SA comme président délégué, avec C. Pras comme administrateur délégué à ses côtés. C. Pras, devenu PDG ensuite, conserve sa position dominante à la faveur des deux souscriptions en numéraires ultérieures, dont l'une réalisée, en 1946, par simple conversion de sommes prélevées sur ses comptes courants.

Il existe donc de multiples façons de prendre le contrôle personnel d'une entreprise de taille moyenne sans en être le fondateur. L'engagement financier requis peut être relativement faible, surtout à la faveur d'une conjoncture économique défavorable comme celle des années 1930. Ces formes de transmission constituent une alternative importan-

(32) Cf. la communication, malheureusement non publiée dans les actes, de V. Labrousse (Université Lyon II) sur Calor au colloque de l'ADHE en 2000 sur les créateurs et créations d'entreprises et les pages qu'elle lui consacre (avec H. Morsel) in M. Lévy-Leboyer, H. Morsel (éd.), Histoire de l'électricité en France, tome 2 : 1919-1946, Paris, Fayard, 1994, p. 1292 et suiv.

(33) Actes du 24 août 1937, 17 juin 1938 et 26 mai 1939, TC Lyon ; ADR, 6U.

(34) Les actions sont cédées pour leur valeur nominale, sans prime d'acquisition.

(35) Cette entreprise, également présente dans le corpus, adopte en 1939 la raison sociale Société lyonnaise des entreprises. L'engagement personnel de C. Pras ne semble pas impliquer de relation de dépendance entre son entreprise d'origine, aux activités de construction électrique sans lien évident, et les Ateliers rhodaniens.

(36) Acte 12 décembre 1938, TC Lyon ; ADR, 6U.

(37) Le fils de l'ancien industriel du tulle et de la dentelle (Dognin \& Cie) et ministre du Commerce Auguste Isaac avait lui-même, en association à l'époque avec un négociant, racheté le fonds en 1912 à son fondateur, Eugène Martin. 
te aux successions familiales. Elles ne les excluent pas cependant, dans la mesure où ces repreneurs peuvent eux-mêmes donner naissance à des dynasties. C'est le cas, par exemple, d'Édouard Brondel: ce fils d'un marinier de la Drôme a racheté en 1912, à 54 ans, le fonds de commerce de « construction mécanique, fonderie de fonte, atelier de menuiserie et de moulage » d'A. Vuillaume à Villeurbanne $^{(38)}$. En 1926, il a préparé sa succession en l'apportant à une SARL qui associe ses trois fils. Après son décès, ce sont eux qui, de manière exclusive, exercent les fonctions dirigeantes, comme gérants puis, après la transformation en SA en 1944, comme PDG. En 1952, un représentant de la troisième génération, Robert (né en 1913), prend les commandes.

De manière générale, la logique familiale apparaît très présente pour la dévolution des fonctions dirigeantes dans le corpus. Ce sont au moins 24 entreprises sur 60 qui peuvent être considérées comme étant sous contrôle familial dominant ${ }^{(39)}$, si ce n'est exclusif, que ce soit au profit des descendants du fondateur (19 cas) ou d'un repreneur ultérieur (5 cas). Même l'entrée en bourse ne l'exclut pas. L'entreprise des Compteurs Garnier, apportée en SA par son fondateur en 1920 et cotée depuis 1931, a eu d'importants actionnaires extérieurs (Cie des compteurs et groupe Durand), mais la famille a conservé jusqu'à la fin de la période la majorité du capital. En 1946, lors de la dernière d'une série de sept augmentations en numéraire intervenues depuis $1931^{(40)}$, Jean Garnier et ses deux enfants souscrivent encore $76 \%$ des actions nouvelles, ce qui représente un engagement de plus de 4,5 millions de francs. Si l'on ajoute les 13 entreprises sous contrôle personnel de leur fondateur ou d'un repreneur, il n'en reste donc qu'un gros tiers (23) à relever d'un contrôle pluriel ou externe. Et, même dans ces cas-là, des formes de survivance d'un contrôle familial existent.

\section{OUVERTURE DU CAPITAL ET SURVIVANCE DE LA LOGIQUE FAMILIALE}

Les modes de contrôle, personnel ou familial, étudiés jusqu'ici étaient à la fois interne - l'actionnaire dominant dirige luimême l'entreprise à titre principal - et exclusif - il n'existait aucun autre actionnaire susceptible de s'opposer à l'influence de l'actionnaire dominant. Deux autres hypothèses doivent être envisagées :

- soit l'entreprise n'est pas contrôlée de manière exclusive par un actionnaire unique ou un groupe d'actionnaires dont les intérêts sont considérés comme confondus (membres d'une même famille), mais par des actionnaires aux intérêts pluriels parmi lesquels aucun ne s'impose de manière dominante (14 cas) ;

- soit elle n'est pas sous le contrôle interne d'actionnaires engagés à titre principal à la direction de l'entreprise, mais sous celui, externe, d'une autre entreprise ou d'un groupe d'entreprises qui délègue sa gestion à des mandataires ( 9 cas).

La première hypothèse trouve souvent son origine dans les conditions de création de l'entreprise, ou au moins de la première société exploitante. Le capital était, dès

(38) Acte 17 juin 1926, TC Lyon ; ADR, 6U.

(39) Un contrôle dominant implique une participation majoritaire ou, éventuellement une importante participation minoritaire qui, face à d'autres intérêts dispersés, ne rencontrerait pas d'opposition.

(40) Elles ont eu lieu en 1923, 1928, 1929, 1931, 1936, 1939 et 1946. Les quatre suivantes d'ici à 1954 s'effectuent sur réserves. 
l'origine, partagé entre plusieurs fondateurs non apparentés entre eux et cette pluralité d'intérêts s'est maintenue ensuite. Le capital du Fil Dynamo (cf. supra) est resté ainsi divisé entre les quatre fondateurs et leurs partenaires extérieurs. Les premiers ont cependant accru leur participation au fil des augmentations en numéraire suivantes, avec des engagements d'environ 53 $\%$ en 1929 et 1943, dont un quart pour le seul André Locquin, PDG jusqu'en 1951. Cette position a suffi à ce dernier pour assurer une continuité familiale. Il est remplacé à la présidence du conseil d'administration par un des investisseurs extérieurs d'origine, Emmanuel Reynier, négociant en fromages à Lyon, mais c'est son gendre Georges Ferrand, ingénieur des Arts et manufactures, qui, en tant qu'administrateur directeur général depuis 1949, lui succède à la direction effective de l'entrepri$\mathrm{se}^{(41)}$. Dans d'autres cas, il y avait bien fondateur unique, mais sa participation ou celle de ses descendants s'est trouvée diluée. Les descendants du fondateur de la Manufacture de caoutchouc industriel Pierre Lacollonge, apportée en SNC par la veuve et le gendre en 1921 puis transformée en SA en 1938, semblent, à la fin de la période, n'avoir plus qu'une position minoritaire au capital. En 1949, à la dernière augmentation en numéraire, la famille, avec la troisième génération en l'occurrence, ne souscrit plus que $5,2 \%$; le reste est apporté par la Banque de l'Indochine et par divers intérêts du caoutchouc indochinois ${ }^{(42)}$. Les héritiers détiennent cependant encore quatre sièges sur sept au conseil d'administration et l'un d'entre eux, Gabriel Simonin (gendre du gendre du fondateur), occupe le poste de PDG. De même, la famille Brachet (figure 2), qui avait repris la fabrique d'appareils de chauffage et de cuisine au gaz fondée par Charles André sous la raison sociale Brachet \& $\operatorname{Richard}^{(43)}$, n'a plus, après la transformation en SA en 1926 et l'entrée en Bourse en 1933, qu'une participation minoritaire : lors des deux augmentations de capital en numéraire suivantes, elle ne souscrit que $18 \%$ en 1944 et $14 \%$ en 1952. Les Brachet se maintiennent cependant aux commandes. Après la deuxième génération avec Maxime Brachet (président puis PDG) et le gendre Alfred Richard (administrateur délégué), la troisième détient ensuite, avec Jacques Brachet, le poste de PDG, au sein d'un conseil d'administration qui comprend six personnalités extérieures à la famille sur huit ${ }^{(44)}$.

(41) G. Ferrand, né en 1921, a épousé Alice Locquin en 1946. Il deviendra PDG de l'entreprise en 1963.

(42) Acte 14 décembre 1949, TC Lyon ; ADR, 6 U.

(43) Alfred Richard est le gendre du repreneur Louis Marie Ernest Brachet.

(44) Charles Pinat, gendre de Maxime Brachet, siège au conseil aux côtés de son beau-frère Jacques Brachet. 
Figure 2 : Descendance de Louis Marie Ernest Brachet (Brachet \& Richard)

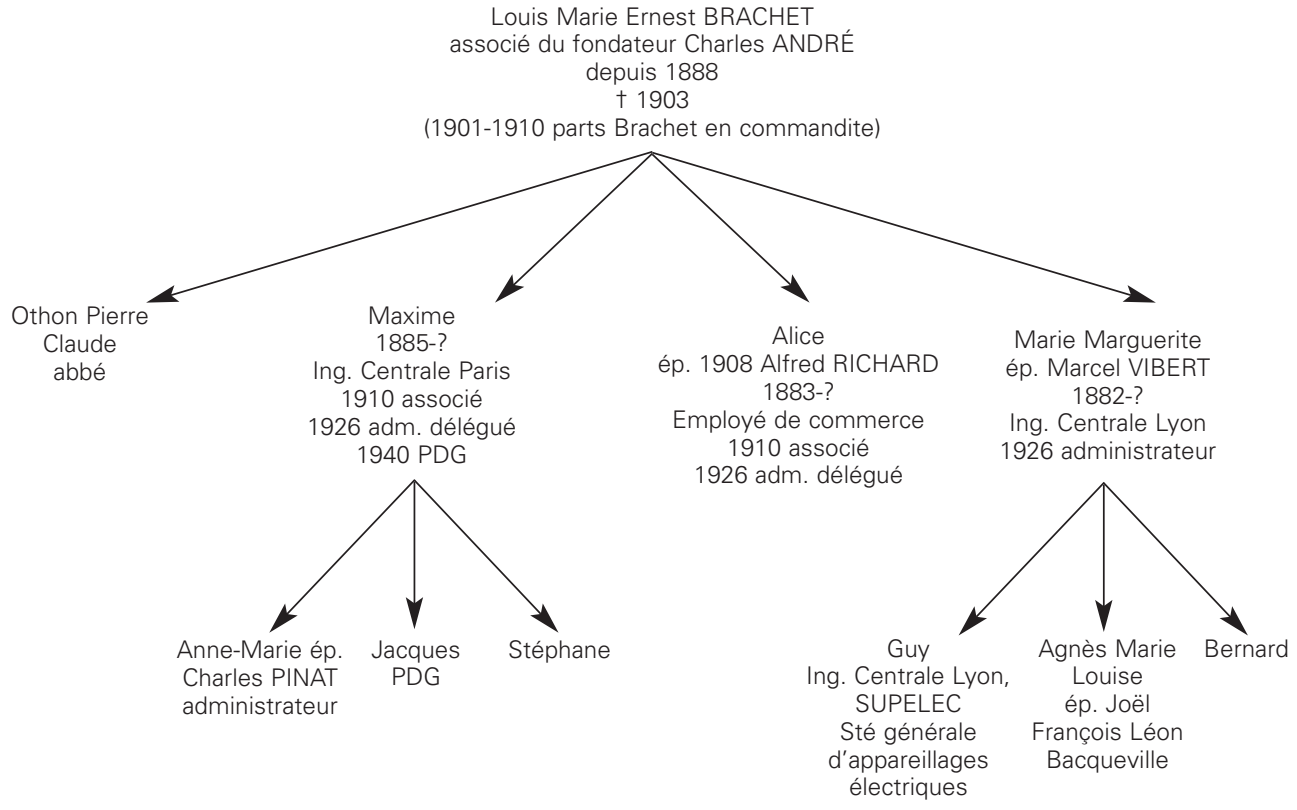

Même lorsque l'influence des familles fondatrices a disparu, les nouveaux dirigeants ne sont pas nécessairement de purs managers. Après la mort de son fondateur Georges Durrschmidt, les établissements éponymes ne sont pas restés très longtemps sous contrôle familial. Le gendre Adolphe Huber a quitté son poste d'administrateur délégué dès 1930 pour se retirer dans sa Suisse d'origine. Les héritiers ne participent plus ensuite que de manière marginale aux augmentations en numéraire. Le successeur Antoine Rougier (administrateur délégué puis PDG) devient en revanche un des principaux actionnaires. À la dernière augmentation de capital en numéraire en 1947 , il est le deuxième souscripteur, avec

$11,1 \%$, contre $2,3 \%$ pour la famille Durrschmidt $^{(45)}$. Mais ce descendant d'une famille de notables lyonnais ${ }^{(46)} n$ 'est pas un manager ordinaire: dès la transformation de l'entreprise en SA en 1921, alors employé âgé de 23 ans seulement, il était, aux côtés de son père Louis (médecin en chef de l'Hôpital chirurgical et de la Polyclinique Saint-Charles), l'un des petits souscripteurs auxquels le fondateur Georges Durrschmidt avait fait appel pour atteindre le minimum légal de 7 actionnaires ${ }^{(47)}$. En 1924, Louis Rougier devient même administrateur de l'entreprise, avant que son fils ne le rejoigne en 1928. À la Société chimique de Gerland, la position d'un Joseph Courbier repose plutôt sur le

(45) Le premier souscripteur est l'industriel apparenté aux Gillet François Balaÿ.

(46) Cf. les nombreuses évocations de la famille Rougier dans C. Pélissier, La vie privée des notables lyonnais, Lyon, Éditions lyonnaises d'art et d'histoire, 1996.

(47) Ils avaient souscrit chacun 25 actions sur un total de 1600. 
prestige de son titre scolaire et sa longévité aux commandes. Ce polytechnicien recruté vers 1908 comme administrateur délégué est déjà en 1920, au milieu d'un capital dispersé, le deuxième souscripteur (avec 4,6\%) d'une augmentation de capital. Devenu ensuite PDG, il installe son fils Jean, également polytechnicien, comme administrateur directeur général. En 1947, ils sont devenus les premiers souscripteurs d'une nouvelle augmentation de capital (avec $16,7 \%$ ). Cette solide position leur permet d'établir une « dynastie managériale».

La deuxième hypothèse, celle d'une entreprise devenue la filiale d'une autre ou d'un groupe, n'exclut pas non plus nécessairement la dimension familiale. En l'absence fréquente de cotation boursière, la prise de contrôle ne peut pas se faire dans l'hostilité aux gérants ou au conseil d'administration en place, dont les statuts requièrent souvent l'agrément pour toute cession de parts ou d'actions à l'extérieur. L'entrée d'un groupe extérieur est destinée à apporter à l'entreprise des moyens nouveaux pour se développer. Elle se fait parfois en plusieurs étapes jusqu'à l'accession à la majorité. Elle n'exclut pas le maintien en fonction des anciens dirigeants. La famille fondatrice de l'entreprise de produits chimiques Gignoux \& Cie à Neuville-surSaône a ainsi commencé en janvier 1947 à céder des parts à des actionnaires extérieurs, dont les intérêts semblent se conjuguer avec ceux de l'Union européenne industrielle et financière, holding du groupe Schneider. Après qu'une majorité du capital se soit trouvée entre leurs mains à la fin 1948, l'entreprise, devenue entre-temps une SA, est restée sous la direction du fils du fondateur, Paul Gignoux, jusqu'à son absorption complète par l'entreprise pharmaceutique
UCLAF en 1953. La situation n'est différente que lorsque l'arrivée d'actionnaires extérieurs intervient de manière brutale, dans un contexte de débâcle financière. Après la liquidation de la société en commandite par actions exploitant la fabrique de produits chimiques agricoles Silvestre \& Cie en 1931, son fondateur et gérant Claude Silvestre disparaît définitivement de l'administration de la nouvelle SA. Ce sont des dirigeants d'Alais, Froges \& Camargue (Pechiney), actionnaire à la hauteur de $69 \%$, qui prennent les commandes.

Parfois, la dimension familiale apparaît aussi à travers l'entreprise repreneuse, lorsqu'elle est elle-même une entreprise familiale. Le capital de l'entreprise lyonnaise de constructions mécaniques pour tissages Verdol est ainsi, lors de sa transformation de SA en SARL en 1946, majoritairement $(50,4 \%)$, aux côtés des descendants du fondateur Jules Verdol, aux mains des deux familles Dalle et Lecomte contrôlant les établissements éponymes, fabricants de matériel pour filature à Bousbecque (Nord). Les entreprises familiales prennent souvent des participations indirectes, par l'intermédiaire de leurs actionnaires ${ }^{(48)}$. La nouvelle gérance reflète le partage du pouvoir entre ancienne et nouvelles familles : d'un côté, la fille et le gendre du fondateur, de l'autre, Antoine Dalle-Lesaffre et Joseph Lecomte. Aux Aciéries du Rhône, suite à la prise de contrôle par les Ets Prénat (Cie de hautsfourneaux et fonderies de Givors), la direction est entièrement aux mains de la famille Prénat $^{(49)}$.

Ce n'est que lorsque la prise de contrôle par une grande entreprise mère, elle-même sous contrôle managérial, est totale que la dévolution des fonctions dirigeantes apparaît

(48) Formant ainsi ce que M. Lescure appelle des groupes informels, centralisés en l'occurrence ; PME et croissance économique. L'expérience française des années 1920, Paris, Économica, 1996, p. 212-218.

(49) Avec Édouard Prénat, arrière-petit-fils du fondateur de la dynastie, comme président et Édouard Charveriat, lié aux Prénat par sa mère, comme directeur général. 
détachée de toute dimension familiale ou patrimoniale. Les présidents successifs installés par Pechiney à la tête de Silvestre sont des responsables salariés de la branche produits chimiques. De même, après la prise de contrôle du constructeur de matériel de travaux publics Pinguely par l'entreprise sidérurgique Châtillon-Commentry en 1946, le poste de PDG est détenu par le directeur général de la seconde, Jean Dupuis. Mais Pinguely ou Silvestre ne sont plus de véritables entreprises autonomes; elles sont devenues, sous la forme de filiales, de simples dépendances d'une grande entreprise.

Dans des entreprises moyennes, l'évolution supposée vers une distanciation croissante entre la propriété et le pouvoir est donc, au moins dans les limites chronologiques de l'étude (1954), souvent très incomplète après plusieurs décennies d'existence. Elle n'est pas nécessairement linéaire, ni même irréversible. Entre les fondateurs encore en place, les héritiers aux commandes, les actionnaires devenus diri- geants ou les dirigeants devenus actionnaires, il reste peu de place pour les purs managers. Par ailleurs, la succession familiale apparaît bien comme la forme dominante de transmission, qu'il s'agisse des dynasties fondatrices, « repreneuses » voire «managériales ». La transmission extrafamiliale, par cession pure et simple du fonds ou des actions, apparaît souvent comme une solution par défaut, en l'absence d'héritiers disponibles. Elle semble souvent relever d'un stade pré-industriel, à un moment où l'entreprise n'a pas encore atteint une dimension qui rend son acquisition inabordable pour un nouveau venu dans les affaires. L'histoire de ces entreprises ne peut pas s'écrire sous le seul angle de considérations économiques: des facteurs personnels tels que le nombre d'enfants en général et de garçons en particulier, leur décalage d'âge par rapport au père et leur éventuelle mortalité, le choix des conjoints pour les filles, la nature des relations familiales en général, apparaissent également déterminants. 